\title{
HIGHLIGHTS
}

MINERAL METABOLISM

\section{Loop diuretics may contribute to secondary hyperparathyroidism}

The investigators of a new study have shown that the use of loop diuretics might be an initiating factor in the early pathogenesis of secondary hyperparathyroidism. "Loop diuretics are known to increase urinary calcium levels and are widely used in chronic kidney disease (CKD). We tested the hypothesis that loop diuretic use would be associated with greater prevalence of secondary hyperparathyroidism," explains researcher Myles Wolf.

\section{4 ...PTH levels were nearly twofold higher in the group receiving loop diuretics than in the untreated group... 77}

A comparison of the effects of loop diuretics and thiazide diuretics on levels of calciuria and parathyroid hormone (PTH), as well as on the prevalence of secondary hyperparathyroidism, was carried out in 3,616 patients with CKD. Primary outcomes were urinary calcium excretion, PTH levels, and presence of secondary hyperparathyroidism (defined as PTH concentration $\geq 65 \mathrm{ng} / \mathrm{l}$ ). In total, 1,083 patients were taking loop diuretics as monotherapy, 723 were on thiazides as monotherapy, 289 were receiving both types of diuretics, and 1,521 were not being treated with diuretics. Baseline characteristics differed between the groups: lower estimated glomerular filtration rate, history of heart failure, increased BMI, and history of diabetes were independent predictors of treatment with loop diuretics, whereas history of hypertension, increasing age, and black race were the strongest predictors of treatment with thiazides.

Crude and adjusted $24 \mathrm{~h}$ urinary calcium excretion and fractional excretion of calcium were highest in the loop diuretic monotherapy group and lowest in the thiazide monotherapy group, whereas serum levels of calcium were lowest in the loop diuretics group and highest in the thiazide group. Median crude PTH levels were nearly twofold higher in the group receiving loop diuretics than in the untreated group; by contrast, thiazide use was associated with modestly reduced mean PTH levels compared with no diuretic use (but only in a model adjusted for case mix). The odds ratio of secondary hyperparathyroidism was 2.1 in loop diuretic users, 0.9 in the thiazide group, and 1.3 in the group taking both types of diuretic (with those on no diuretics as the reference group with an odds ratio of 1). "Alone, thiazides did not alter risk of secondary hyperparathyroidism, but patients with CKD stages 2-3 (but not stage 4) treated with both loop diuretics and thiazides were protected from the tendency of loop diuretics to associate with secondary hyperparathyroidism," says Wolf.

Known mechanisms of secondary hyperparathyroidism in CKD include hyperphosphatemia, hypocalcemia and calcitriol deficiency. Loop diuretics might contribute to the disease pathogenesis by increasing calciuria. "Treatments such as thiazides, which attenuate calciuria, might be beneficial in patients who need loop diuretics. Alternatively, administering calcitriol to patients receiving loop diuretics might attenuate the severity or delay the onset of secondary hyperparathyroidism," notes Wolf.

Helene Myrvang

Original article Isakova, T. et al. Diuretics, calciuria and secondary hyperparathroidism in the Chronic Renal Insufficiency Cohort. Nephrol. Dial. Transplant. doi:10.1093/ndt/gfr026 